\title{
A Flat Elevated Lesion of Well-differentiated Adenocarcinoma with Lymphoid Stroma of the Colon
}

Hideaki Harada $^{1 *}$, Satoshi Suehiro ${ }^{1}$, Takanori Shimizu${ }^{1}$, Masatoshi Hashimoto ${ }^{2}$, Yasushi Katsuyama ${ }^{1}$ and Kenji Hayasaka ${ }^{1}$

${ }^{1}$ Department of Gastroenterology, New Tokyo Hospital, Chiba 270-2232, Japan

${ }^{2}$ Department of Surgery, New Tokyo Hospital, Chiba 270-2232, Japan

\begin{abstract}
Here, we present a rare case of a 78-year-old male with well-differentiated adenocarcinoma with lymphoid stroma of the colon. Colonoscopy revealed a type 0-lla-like lesion located at the descending colon. We performed endoscopic submucosal dissection of the lesion. Histological examination of the resected specimen revealed that the tumor was comprised of well-differentiated adenocarcinoma with lymphoid cell infiltration accompanied by submucosal lymphoid follicle formation. Because of a positive for vertical resection margin, we performed an additional surgical resection. However, we found no residual tumor or lymph node metastases. To the best of our knowledge, this is a rare report describing a case of dome-type carcinoma with lymphoid stroma of the colon for which magnifying endoscopy with crystal violet staining and narrow-band imaging was performed.
\end{abstract}

Keywords: Lymphoid stroma; Well differentiated adenocarcinoma; Limphoepithelioma-like carcinoma; Dome-type carcinoma; Magnifying endoscopy; Narrow-band imaging; Endoscopic submucosal dissection

\section{Introduction}

Colorectal cancer presenting as submucosal tumor (SMT) is relatively rare, and colorectal cancer presenting as SMT with lymphoid stroma is rare. Several cases of lymphoepithelioma-like carcinoma (LELC) have been reported. LELC of the colon is histologically poorly differentiated and characterized by ill-defined borders, prominent stroma, intratumoral lymphoid infiltrate and a syncytial growth pattern [1-3]. On the other hand, there have been some reports of welldifferentiated adenocarcinoma with lymphoid stroma of the colon, the so-called dome-type carcinoma (DC) [4-7].

To our knowledge, this is a rare report of DC with lymphoid stroma of the colon for which magnifying endoscopy (ME) with crystal violet staining and Narrow-band imaging (NBI) was performed.

\section{Case Report}

The patient was a 78-year-old male who underwent a colonoscopy at follow-up after surgery for sigmoid colonic cancer. Colonoscopy revealed a type 0-IIa-like lesion located at the descending colon (Figure 1A). Conventional endoscopy revealed a reddish, slightly elevated lesion. Spraying with indigo-carmine solution revealed a well-demarcated 0-IIa-like lesion (Figure 1B). ME with NBI revealed a surface of glandular structures relatively uniform in size and without abnormal microvessels (Figure 1C). ME after staining with crystal violet revealed a type II (Kudo's classification) pit pattern surface and a type $\mathrm{Vi}$ pit pattern in the center of the lesion [4] (Figure 1D).

To determine malignancy, we obtained written informed consent from the patient and performed endoscopic submucosal dissection (ESD). Although the submucosal layer was slightly fibrotic during the submucosal dissection, en bloc resection of the lesion was successfully performed (Figures $2 \mathrm{~A}$ and $2 \mathrm{~B}$ ). The post-procedural course was uneventful without bleeding and perforation.

Histological examination of the resected specimen (measuring $23 \times 16 \mathrm{~mm}$; Figure $2 \mathrm{C}$ ) revealed that the tumor was mostly covered with normal epithelium and comprised of well-differentiated adenocarcinoma with lymphoid cell infiltration accompanied by submucosal lymphoid follicle formation (Figures 3A and 3B). Some of

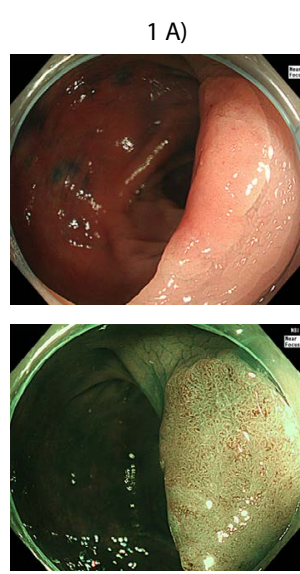

$1 \mathrm{C})$
$1 \mathrm{~B})$

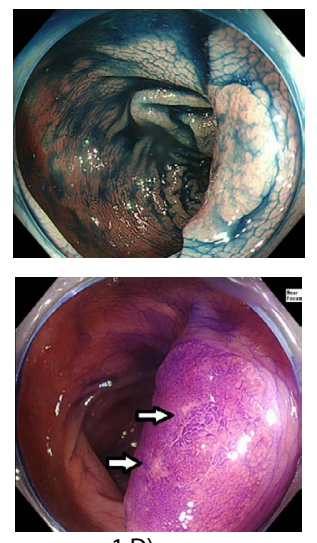

1 D)
Figure 1: A. Conventional endoscopy revealed a reddish, elevated lesion approximately $20 \mathrm{~mm}$ in diameter. B. Chromoendoscopic view with indigocarmine solution revealed a well-demarcated lesion margin. $\mathbf{C}$. The surface of the lesion was observed by ME with NBI to be glandular structures of relatively uniform size without abnormal microvessels. D. Chromoendoscopic view of the surface with crystal violet staining revealed a type II pit pattern and partially a type Vi pit pattern in the center of the lesion (white arrow) (Kudo's classification).

the glands were dilated as cystic. EBV-related latent membrane protein (LMP) - 1 was negative (Figure 3C). The resected tumor was positive for vertical resection margin. Therefore, an additional surgical resection was performed, and there were no residual tumor or lymph node metastases in the surgical specimen (Figures $4 \mathrm{~A}-4 \mathrm{C}$ ). The patient was recurrence-free after a 12-month follow up.

*Corresponding author: Hideaki Harada, Department of Gastroenterology, New Tokyo Hospital, Chiba, Japan, 1271 Wanagaya, Matsudo Chiba, Japan, Tel: +8147-711-8700; Fax: +81-47-392-8718; E-mail: nerimaendo@hotmail.co.jp

Received October 28, 2015; Accepted December 01, 2015; Published December 03, 2015

Citation: Harada H, Suehiro S, Shimizu T, Hashimoto M, Katsuyama Y, et al. (2015) A Flat Elevated Lesion of Well-differentiated Adenocarcinoma with Lymphoid Stroma of the Colon. J Cytol Histol S3:026. doi:10.4172/2157-7099.S3-026

Copyright: ( 2015 Harada $\mathrm{H}$, et al. This is an open-access article distributed under the terms of the Creative Commons Attribution License, which permits unrestricted use, distribution, and reproduction in any medium, provided the original author and source are credited. 

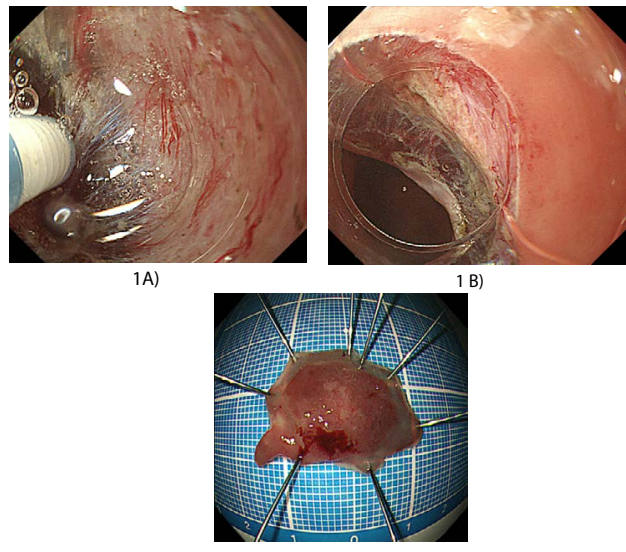

$1 \mathrm{C)}$

Figure 2: Endoscopic submucosal dissection of the lesion. A. Slightly fibrotic submucosal dissection with Flush Knife BT. B. An artificial ulcer observed after lesion removal. C. The resected specimen measured $23 \times 16 \mathrm{~mm}$.
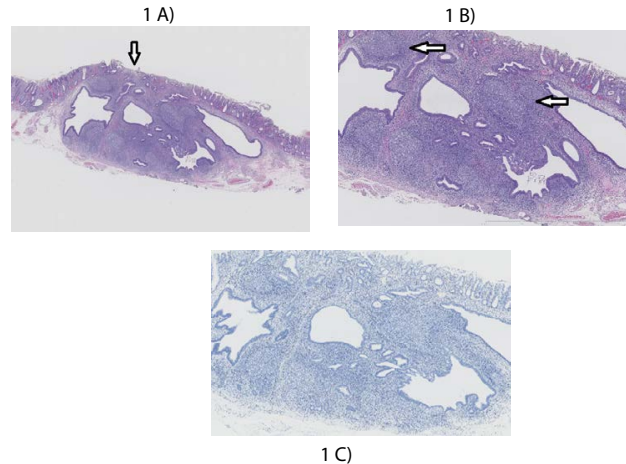

Figure 3: A. The submucosal layer structure appeared as a well-differentiated adenocarcinoma with lymphoid stroma, hematoxylin and eosin (H\&E) stain; loupe. B. Cystically dilated glands and lymphoid follicle formation (white arrow) located in the submucosa, H\&E stain; magnification $4 \mathrm{X}$. C. Immunohistochemical staining of resected specimen showed it was negative for EBV, LMP-1; magnification $4 \mathrm{X}$.

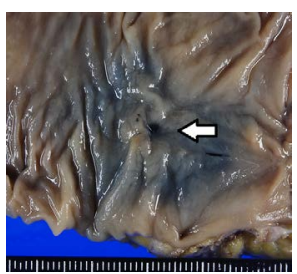

1 A)

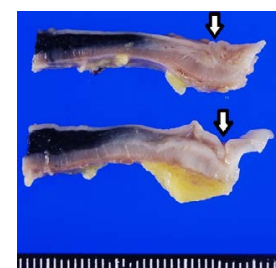

$1 \mathrm{~B})$

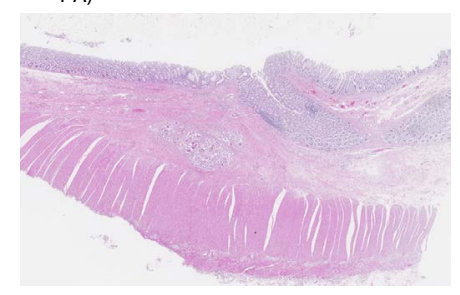

$1 \mathrm{C})$

Figure 4: Surgically resected specimen. A. A scar after endoscopic resection can be seen at the white arrow. B. The transected image of the resected specimen. A scar and a fibrotic formation can be seen at the white arrow. C. Histopathological examination shows no residual tumor in the surgical specimen.

\section{Discussion}

Colorectal cancer presenting as SMT with lymphoid stroma is rare. In this case, although the histological examination was welldifferentiated adenocarcinoma, lymphoid cell infiltration accompanied by lymphoid follicle formation was seen in the submucosal layer. Jass et al. reported on a patient with a well-differentiated adenocarcinoma of the colon as DC with lymphoid stroma [5]. DC represents endoscopic and pathological findings as SMT, namely, a well- and/or moderatelydifferentiated adenocarcinoma with lymphoid stroma and dilated tumor glands in the submucosal layer [5]. In this case, the surface of the tumor can be covered with normal epithelium, however, if a depression or an erosion in the center of the tumor is present, it might be a denudation of the tumor from the submucosal layer. ME after staining with crystal violet revealed a type Vi pit pattern as erosions in the center of the tumor (Figure 1D, white arrow). The denudation of the tumor was recognizable through the resected specimen by ESD (Figure 3A). In case of suspected colorectal cancer presenting as SMT, checking whether a depression or an erosion is present in the center of the tumor is important for identifying DC.

Although Epstein-Barr virus (EBV) has been implicated in previous LELC colon studies [8], in this case, immunohistochemical staining of the resected specimen was EBV-negative. To our knowledge, there have been no reports on DC with EBV infection. Also, DC does not feature microsatellite instability. The lack of evidence for microsatellite instability and EBV infection is consistent with the concept that lymphoid infiltration associated with DC reflects the nature of its tissue of origin, which is the dome epithelium [7].

Mori et al. reported on a patient with LELC of the colon treated with ESD [9]. To our knowledge, there are few cases of colorectal cancer presenting as SMT treated with ESD. The same as in Mori's case, we performed ESD for dome-like carcinoma. The tumor was negative for lateral resection margin without lymphatic metastasis. However, it was positive for vertical resection margin. As DC tumor itself present in the submucosal layer, endoscopic treatment for DC is controversial. However, we believe that diagnostic resection of DC endoscopically is well indicated in suspected cases of early-stage DC.

\section{Conclusion}

This is a rare case of well differentiated adenocarcinoma with lymphoid stroma of the colon for which ME with crystal violet staining and NBI was performed.

\section{References}

1. Vilor M, Tsutsumi Y (1995) Localization of Epstein-Barr virus genome in lymphoid cells in poorly differentiated adenocarcinoma with lymphoid stroma of the colon. Pathol Int 45: 695-697.

2. lezzoni JC, Gaffey MJ, Weiss LM (1995) The role of Epstein-Barr virus in lymphoepithelioma-like carcinomas. Am J Clin Pathol 103: 308-315.

3. Matsunou H, Konishi F, Hori H, Ikeda T, Sasaki K, et al. (1996) Characteristics of Epstein-Barr virus-associated gastric carcinoma with lymphoid stroma in Japan. Cancer 77: 1998-2004.

4. Kudo S, Hirota S, Nakajima T, Hosobe S, Kusaka H, et al. (1994) Colorectal tumours and pit pattern. J Clin Pathol 47: 880-885.

5. Jass JR, Constable L, Sutherland R, Winterford C, Walsh MD, et al. (2000) Adenocarcinoma of colon differentiating as dome epithelium of gut-associated lymphoid tissue. Histopathology 36: 116-120.

6. Takagi W, Yamamoto K, Amano T, Sakamoto A, Otake Y, et al. (2015) Colon adenocarcinoma with dome-like phenotype: characteristic endoscopic ultrasonography (EUS) findings. Endosc Int Open 3: E359-362. 
Citation: Harada H, Suehiro S, Shimizu T, Hashimoto M, Katsuyama Y, et al. (2015) A Flat Elevated Lesion of Well-differentiated Adenocarcinoma with Lymphoid Stroma of the Colon. J Cytol Histol S3:026. doi:10.4172/2157-7099.S3-026

Page 3 of 3

7. Yamada M, Sekine S, Matsuda T, Yoshida M, Taniguchi H, et al. (2012) Dometype carcinoma of the colon; a rare variant of adenocarcinoma resembling a submucosal tumor: a case report. BMC Gastroenterol 8: 12: 21

8. Kon S, Kasai K, Tsuzuki N, Nishibe M, Kitagawa T, et al. (2001)
Lymphoepithelioma-like carcinoma of rectum: possible relation with EBV. Pathol Res Pract 197: 577-582.

9. Mori Y, Akagi K, Yano M, Sashiyama H, Tsutsumi O, et al. (2013) Lymphoepithelioma-like carcinoma of the colon. Case Rep Gastroenterol 7 : 127-133.

This article was originally published in a special issue, Cytopathology handled by Editor(s). Borislav A. Alexiev. Department of Pathology University of Maryland Medical Center, USA 